\title{
ELDERLY PATIENTS
}

\section{Surgical mortality in patients more than 80 years of age}

\author{
AM Khan-Kheil, HN Khan
}

University Hospital Coventry \& Warwickshire NHS Trust, UK

\section{ABSTRACT}

INTRODUCTION Patients aged $>80$ years account for a considerable proportion of the population admitted to hospital under general surgeons. We aimed to establish the prevalence of mortality in patients aged $>80$ years who underwent emergency general, vascular and urological surgery within a 13-month period at a large teaching hospital in the UK.

MATERIALS AND METHODS A retrospective analysis was carried out of all patients aged $\geq 80$ years admitted on acute on-call emergency under general, vascular or urological surgeons. Patient demographics (including co-morbidities and sex) were recorded. American Society of Anesthesiologists scores were reviewed from anaesthetic records. The outcome measure was 30-day mortality for those who had undergone emergency general, vascular or urological surgery.

RESULTS A total of 4,069 patients were admitted under general, vascular and urological surgeons during the study period. Of these patients, 521 were aged $>80$ years. Sixty-three patients underwent emergency surgery and 12 died $<30$ days after surgery (mortality $=19 \%$ ).

The most common procedure was laparotomy (20 cases). The most common co-morbidity was cardiac disease, which included hypertension, ischaemic heart disease, and hypercholesterolemia. A considerable proportion of patients also had malignant disease and arthritis.

CONCLUSIONS The present study suggests that emergency surgery should not be denied to subjects aged $>80$ years based on age alone. Mortality varies according to the type of emergency procedure. Mortality was highest after laparotomy and vascular surgery whereas, for more routine procedures such as hernia repair and abscess drainage, survival was almost $100 \%$ after 30 days.

\section{KEYWORDS}

Elderly - Mortality - Co-morbidity - Survival

Accepted 14 March 2015

CORRESPONDENCE TO:

Ayisha Mehtab Khan-Kheil, E: a.khan-kheil@nhs.net

In a recent report from the Royal College of Surgeons of England highlighting challenges for the surgical profession, emergency surgery was a key issue. Mortality in those aged $>80$ years was reported to be $>25 \%$, varying 12 fold between the best- and worst-performing hospitals. ${ }^{1}$

Patients aged $>80$ years now account for a considerable proportion of the population admitted to hospital under general surgeons. In the UK, 3 million people fall into this age group, and this number is expected to double by $2030 .^{2}$

We hypothesised that, despite advanced age and multiple co-morbidities, offering emergency surgery to this group of patients (if indicated) is reasonably safe. Therefore, we aimed to establish the prevalence of mortality in patients aged $>80$ years who underwent emergency general, vascular or urological surgery within a 13-month period at a large teaching hospital in the UK.

\section{Methods}

A retrospective analysis was carried out of all patients aged $\geq 80$ years admitted on acute on-call emergency under general, vascular or urological surgeons. The study period was 1 July 2012 to 31 July 2013 (inclusive). Only the case notes of all appropriately aged patients undergoing surgery during acute admission were assessed. Patient demographics (including co-morbidities and sex) were recorded. Scores based on the classification system for physical status set by the American Society of Anesthesiologists (ASA) were reviewed from anaesthetic records. The outcome measure was 30-day mortality for those who had undergone emergency general, vascular or urological surgery. The information was input onto Microsoft Excel 2008 and analysed accordingly.

\section{Results}

A total of 4,069 patients were admitted under general, vascular or urological surgeons during the study period. Of these patients, 521 were aged $>80$ years. Sixty-three patients (34 males and 29 females; mean age, 84.5 years) underwent emergency surgery and 12 died $<30$ days after surgery $($ mortality $=19 \%)$. 
Table 1 Procedure type and 30-day mortality in patients aged $>80$ years undergoing emergency surgery

\begin{tabular}{|c|c|c|c|c|}
\hline Procedure & $\begin{array}{l}\text { Number of } \\
\text { patients }\end{array}$ & $\begin{array}{l}\text { Alive after } \\
30 \text { days }\end{array}$ & $\begin{array}{l}\text { Deceased } \\
\text { after } 30 \text { days }\end{array}$ & Mortality (\%) \\
\hline Laparotomy & 20 & 15 & 5 & $25 \%$ \\
\hline Obstruction/resection of the small bowel & 8 & 5 & 3 & \\
\hline Hartmann procedure & 5 & 3 & 2 & \\
\hline Strangulated hernia & 2 & 2 & 0 & \\
\hline Other & 5 & 5 & 0 & \\
\hline Urological & 5 & 4 & 1 & $20 \%$ \\
\hline Incision and drainage of abscess & 8 & 7 & 1 & $12.5 \%$ \\
\hline Hernia repair & 13 & 13 & 0 & $0 \%$ \\
\hline Femoral & 2 & 2 & 0 & \\
\hline Inguinal & 6 & 6 & 0 & \\
\hline Para-umbilical & 5 & 5 & 0 & \\
\hline Vascular & 12 & 8 & 4 & $33.3 \%$ \\
\hline Critical limb ischaemia necessitating embolectomy/bypass & 6 & 4 & 2 & \\
\hline Above-knee amputation & 1 & 0 & 1 & \\
\hline Below-knee amputation & 5 & 4 & 1 & \\
\hline Other & 5 & 4 & 1 & $20 \%$ \\
\hline Total & 63 & 51 & 12 & $19 \%$ \\
\hline
\end{tabular}

Table 1 denotes the type of surgery undertaken and survival at 30 days. The most common procedure was laparotomy (20 cases). Other procedures were: vascular; incision and drainage of abscesses; hernia repair; and urological procedures.

Figure 1 demonstrates the co-morbidities of all those undergoing emergency surgery. The most common comorbidity was cardiac disease, which included hypertension, ischaemic heart disease, and hypercholesterolemia. A considerable proportion of patients also had malignant disease and arthritis.

\section{Discussion}

Surgical mortality in the elderly is controversial. However, due to an aging population as well as advancements in surgical procedures and postoperative care, surgery in this age group is becoming more common.

From our data collected over 13 months, there were 4,069 admissions to the surgical admissions unit of a teaching hospital. Of these admissions, 521 of patients were aged $>80$ years $(13 \%)$. We established that 30 -day mortality after emergency surgery in this age group was $19 \%$. However, this figure takes into account all types of emergency surgery that was carried out, ranging from laparotomy to hernia repair to vascular procedures. Similar studies looking at surgical outcomes in this age group state that, in common procedures such as hernia repair, 30-day mortality can be $<2 \%$ but, if postoperative complications occur, then mortality can increase to $33 \% .^{3}$

Several factors predispose to postoperative complications in this age group: pre-existing disease, ASA >III, and the need for a major surgical procedure. ${ }^{4}$

In the present study, the ASA scores of the 12 patients who died were $>$ III, suggesting that poor preoperative status led to an unfavourable postoperative outcome. Also, 55 of the 63 patients who underwent emergency surgery had cardiac co-morbidities (most commonly hypertension). However, also included in this category were ischaemic heart disease, atrial fibrillation and previous myocardial infarction. Other prevalent co-morbidities were arthritis (which would be expected in this age group) but also 


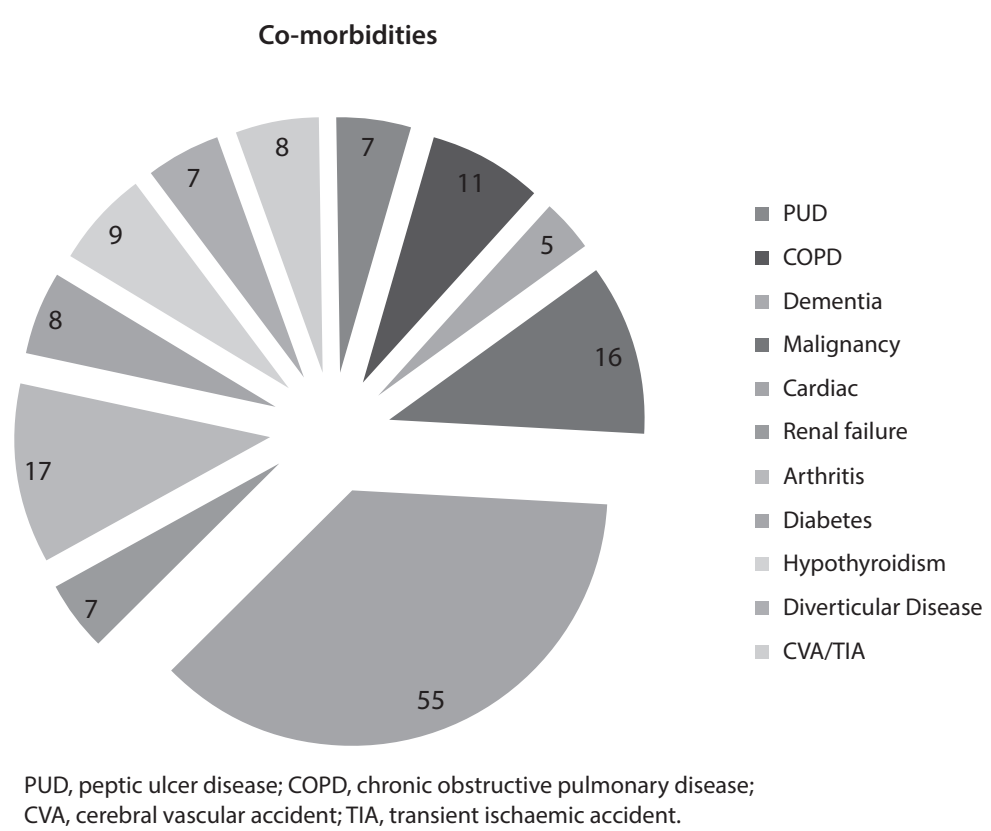

Figure 1 Comorbidities in the study cohort

malignant disease. The latter was mainly colorectal malignancy, hence the need for emergency laparotomy due to bowel obstruction.

Hessman et al looked at survival in elderly patients with colorectal cancer. They concluded that age should not be a disqualifying factor for surgery. They also reported that the ASA score was a better predictor of perioperative mortality and survival than age alone. ${ }^{5}$ ASA score is a subjective assessment of physical health based on five classes. Class III denotes severe systemic disease. ${ }^{6}$ In relation to postoperative mortality in emergency surgery, ASA $=$ I reflects a $1 / 1000$ chance of postoperative complications, whereas ASA $\geq \mathrm{IV}$ denotes a 26.5/1000 risk. $^{7}$

In 2003, Abbas and Booth investigated mortality after major abdominal surgery in octogenarians. After emergency surgery, 30-day mortality was $29 \%$, yet only $7.5 \%$ after elective procedures. ${ }^{8}$ Also, patients with ASA $=\mathrm{IV} / \mathrm{V}$ were less likely to survive than those with ASA $=$ II/III. This finding is in accordance with our study in which $>70 \%$ of those who underwent emergency surgery had ASA $>$ III (including 11 out of the 12 patients who died). Mortality in the study by Abbas and Booth appears to be higher than ours, but their study focused only on abdominal surgery. Furthermore, long-term survival was similar regardless of whether surgery was emergency or elective when compared with an age-matched population.

Various studies carried out in the UK have demonstrated a similar prevalence of mortality in this age group. In 2012, Saunders et al investigated variations in mortality after emergency laparotomy across 35 National Health Service Hospitals. Results showed that mortality in all age groups was $14.9 \%$, and was $24.4 \%$ in those aged $\geq 80 .{ }^{9}$ ASA score was a better predictor of postoperative mortality, and ASA $\geq$ III was classified as 'high risk'. Mortality was also affected by whether the patient was transferred to the ward or to the intensive care unit postoperatively, suggesting that surgical mortality is dependent on the ability of clinicians to recognise and manage surgical complications. ${ }^{10}$

Byrne et al. highlighted that a considerable burden of mortality lies outside the 30-day 'window', and suggested that 90-day mortality should be considered. In their study, mortality after colorectal surgery was $8.5 \%$ after 30 days and $11.3 \%$ after 90 days. ${ }^{11}$ They also stated that mortality was greater with advancing age and increasing number of co-morbidities.

A recent review looking at 17 studies of elderly patients undergoing elective colorectal resection highlighted 30mortality of $0-13 \% .^{12}$ However, that study also questioned the value of recording 30-day mortality in the elderly because mortality can remain $\leq 1 \%$ even 1 year after surgery.

\section{Conclusion}

The present study suggests that emergency surgery should not be denied to subjects aged $>80$ years based on age alone. Mortality varies according to the type of emergency procedure. In our study, mortality was highest after laparotomy and vascular surgery whereas, for more routine procedures such as hernia repair and abscess drainage, survival was $100 \%$ after 30 days.

Our study had limitations. Firstly, we focused only on patients who had a surgical intervention. Hence, we could not comment on patients who did not undergo surgery. Secondly, the study cohort was small: only 63 patients had 
emergency procedures. Thirdly, as noted by other scholars, 30 -day mortality in individuals aged $>80$ years may not be the most accurate way to assess survival. Hence, if we had employed a much longer follow-up, more accurate survival data could have been obtained. Finally, it is important to note that our study carried out at a single university hospital. Comparison of outcomes from smaller district general hospitals would have enabled a more general overview of emergency surgical outcomes in this age group. Thus, though we deem it reasonably safe for emergency surgical procedures in those aged $>80$ years to be carried out at our hospital, this may not be the case for smaller hospitals in terms of postoperative recovery, beds in the intensive therapy unit, and access to emergency imaging.

Overall, we have shown that age should not be a disqualifying factor for emergency surgery in those aged $>80$ years. Most patients who underwent surgery had ASA $\geq \mathrm{III}$ and multiple co-morbidities, but the majority survived 30 days after surgery. Of the patients who died, many had poor functional status preoperatively, and surgery may have been a 'last resort' that failed. Further work is needed on a larger cohort over a longer period of time, but these data are useful additions to the evidence base.

\section{References}

1. Horrocks M. Current challenges for surgery. Available from www.rcseng.ac.uk/ surgeons/supporting-surgeons/regional/docs/mike-horrocks-session-1 (accessed 15 September 2015).
2. United Kingdom Parliament. The ageing population: key issues for the 2010 Parliament. Available from:www.parliament.uk/business/publications/research/ key-issues-for-the-new-parliament/value-for-money-in-public-services/the-ageingpopulation/ (accessed 15 September 2015).

3. Hamel MB, Henderson WG, Khuri SF et al. Surgical outcomes for patients aged 80 and older: morbidity and mortality from major noncardiac surgery. J Am Geriatr Soc 2005; 53: 424-429.

4. Jin F, Chung F. Minimizing perioperative adverse events in the elderly. $\mathrm{Br} J$ Anaesth 2001; 87: 608-624.

5. Hessman O, Bergkvist L, Strom S. Colorectal cancer in patients over 75 years of age - determinants of outcome. Eur J Surg Oncol 1997; 23: 13-19.

6. Daabiss M. American Society of Anaesthesiologists physical status classification. Indian J Anaesth 2011; 55: 111-115.

7. Tiret L, Hatton F, Desmonts JM, Vourc'h G. Prediction of outcome of anaesthesia in patients over 40 years: a multifactorial risk index. Stat Med 1988; 7: 947-954.

8. Abbas S, Booth M. Major abdominal surgery in octogenarians. N Z Med J 2003; 116: U402.

9. Saunders DI, Murray D, Pichel AC, Varley S, Peden CJ; UK Emergency Laparotomy Network. Variations in mortality after emergency laparotomy: the first report of the UK Emergency Laparotomy Network. Br J Anaesth 2012; 109: 368-375.

10. Ghaferi AA, Birkmeyer JD, Dimick JB. Variation in hospital mortality associated with inpatient surgery. N Engl J Med 2009; 361:1,368-1,375.

11. Byrne BE, Mamidanna R, Vincent CA et al. Population-based cohort study comparing 30- and 90-day institutional mortality rates after colorectal surgery. Br J Surg 2013; 100: 1,810-1,817.

12. Mamidanna R, Almoudaris AM, Faiz 0 . Is 30-day mortality an appropriate measure of risk in elderely patients undergoing elective colorectal resection? Colorectal Dis 2012; 14: 1,175-1,182. 